\title{
Tako-tsubo cardiomyopathy as a recurrent disease with doubtful prognosis of recovery and heterogenic symptoms
}

\author{
Grzegorz Kubiak ${ }^{1}$, Wojciech Jacheć ${ }^{1}$, Damian Kawecki ${ }^{1}$, Magdalena Traczewska ${ }^{2}$, \\ Grzegorz Irlik ${ }^{1}$, Ewa Nowalany-Kozielska ${ }^{1}$ \\ ${ }^{1} 2^{\text {nd }}$ Department of Cardiology, Silesian Medical University, Zabrze, Poland \\ ${ }^{2}$ Department of Radiology, Silesian Medical University, Zabrze, Poland
}

\begin{abstract}
Tako-tsubo cardiomyopathy, known since 1990, is described as hypo/akinesis of apical heart segments with the hyperkinesis of the basis of the heart which mimics the shape of a traditional Japanese octopus trap. (Cardiol J 2012; 19, 5: 521-523)
\end{abstract}

Key words: tako-tsubo, apical ballooning syndrome, myocardial stunning

\section{Introduction}

Tako-tsubo cardiomyopathy, known since 1990 , is described as hypo/akinesis of apical heart segments with the hyperkinesis of the basis of the heart which mimics the shape of a traditional Japanese octopus trap [1]. It occurs predominantly in women after the menopause and is induced by emotional or exogenous stress, for example: surgical procedure, hypertension, sudden onset of pain. Clinical symptoms consist of chest pain with electrocardiographic features of ST elevated acute coronary syndrome (ST elevation in leads V3-V6, II, III, aVL, aVF, V1) and usually moderate increase of myocardial necrosis markers. In only $10 \%$ of cases, clinical symptoms can mimic NSTE-ACS [2, 3, 4-8]. Unlike in typical ACS, there are no atherosclerotic lesions in coronary arteries (Fig. 1). Takotsubo cardiomyopathy is also known as apical ballooning syndrome (ABS). It is believed to be triggered by catecholamine stimulation of $\mathrm{B} 1$ and $\mathrm{B} 2$ receptors which causes decreased expression of regulator proteins SERCa2a and PLN depending on $\mathrm{Ca} 2+[9]$. Most frequently, myocardial stunning affects the apical segments of the heart which is caused by higher affinity to adrenaline. In a typical case of tako-tsubo cardiomyopathy, elevation of ST segment in the ECG lasts usually three days from the sudden onset of angina, then $T$ waves inversion and QT extension can be observed. Contractility dysfunction regresses after up to 30 days $[2,3,10]$. We present two case reports of recurrent tako-tsubo cardiomyopathy without elevation of ST segments in the ECG. It is worth noting that in the second presented case, onset of the disease occurred more than ten years after the first manifestation. Recurrence is claimed only in $10 \%$ of all cases of tako-tsubo cardiomyopathy [1-3].

\section{Case history 1}

A 59 year-old woman with intermittent chest pain of four days' duration was admitted to our ward with a diagnosis of NSTE-ACS. She had a history of DDDR implantation in 1999 due to atrio-ventricular (A-V) block type II. The initial ECG showed atrial stimulation with normal A-V conduction $60 / \mathrm{min}$, T wave inversion in leads: I, aVL, V3-V6. Serum levels of troponin I were $0.206 \mu \mathrm{g} / \mathrm{L}(\mathrm{N}<0.030 \mu \mathrm{g} / \mathrm{L})$. Coronary angiography did not reveal relevant coronary artery stenosis. Echocardiographic examination showed no contractility disturbances with nor-

Address for correspondence: Grzegorz Kubiak, MD, $2^{\text {nd }}$ Department of Cardiology, Silesian Medical University, ul. M. Curie-Skłodowska, 41-800 Zabrze, Poland, e-mail: greg_kubiak@yahoo.com 


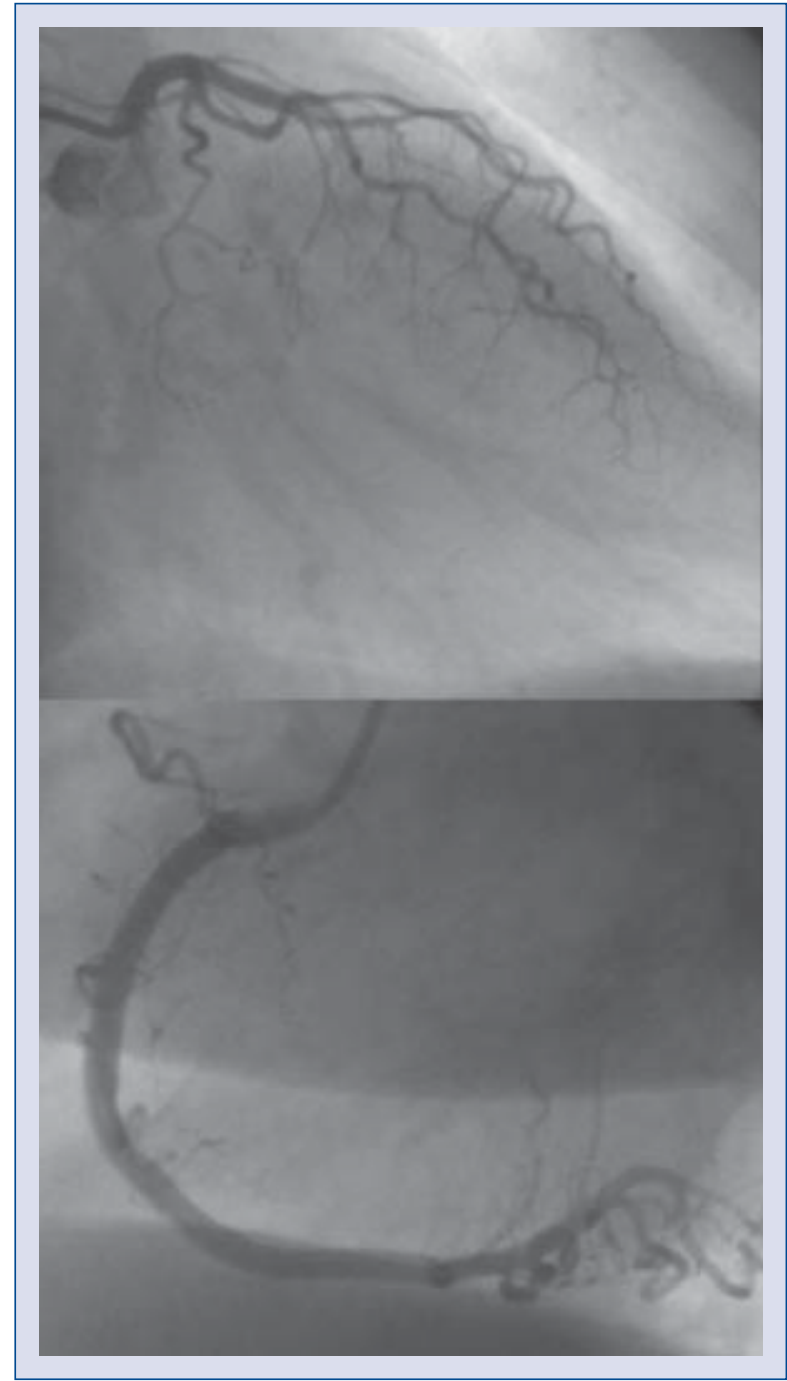

Figure 1. No significant lesions in coronary arteries.

mal left ventricular ejection fraction (LVEF) of $60 \%$. The patient has been treated in our hospital in 1999 due to sudden onset of chest pain related to emotional stress before a tonsillectomy procedure. In echocardiography, ejection fraction reduced to $25 \%$ with typical hypo-kinesis of apical segments, and dilation of left ventricular up to $150 \mathrm{~mL}$ was then observed. Control examination after ten days showed augmentation of ejection fraction up to $60 \%$ with total regression of contractility disturbances (Figs. 2, 3).

\section{Case history 2}

A 74 year-old woman presenting with typical chest pain was admitted to our ward with a diagnosis of NSTE-ACS. The initial ECG showed T wave inversion in III, aVF, V1-V6. In echocardiography, typical for tako-tsubo cardiomyopathy contractility

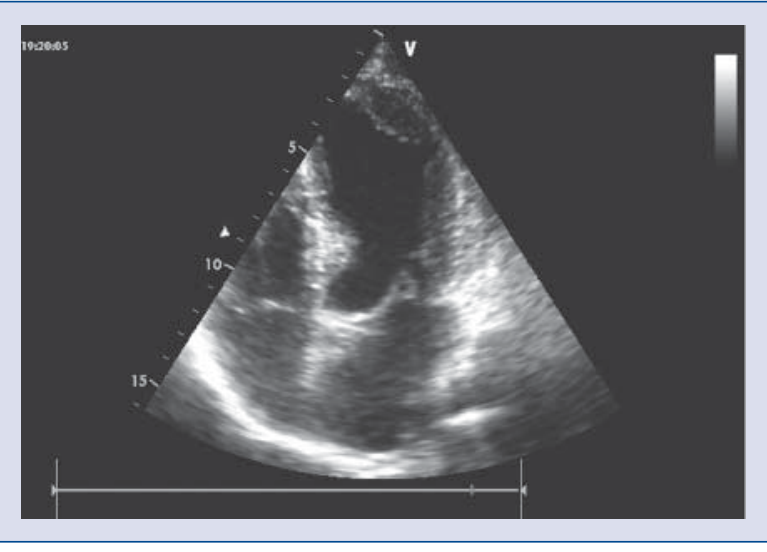

Figure 2. Typical left ventricle contractility disturbances during acute phase of the disease.

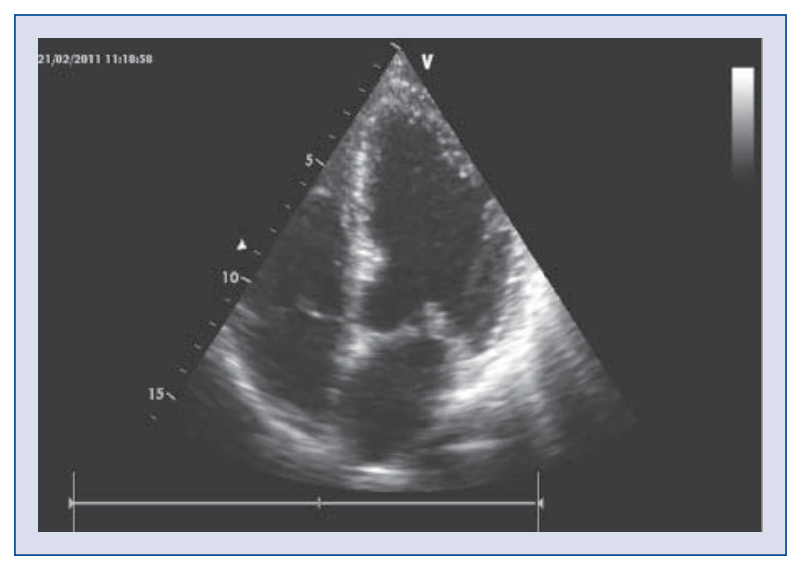

Figure 3. Normal left ventricle contractility three days after symptoms onset.

disturbances with a LVEF of $40 \%$ were observed. Serum levels of CK-MB were $91 \mathrm{IU} / \mathrm{L}(\mathrm{N}<25.0 \mathrm{IU} / \mathrm{L})$ and troponin I was $1,639 \mathrm{ng} / \mathrm{L}(\mathrm{N}<14 \mathrm{ng} / \mathrm{L})$. In the coronary angiography, no relevant coronary artery stenosis was diagnosed. After ten days, LVEF had increased to $50 \%$. It is worth noting that the same patient had been examined due to stable angina (including coronary angiography with no relevant stenosis and proper echocardiographic examination) six months before the NSTE-ACS occurred.

\section{Discussion}

Apical segment of left ventricle is predominantly affected by hypo/akinesia due to its higher affinity to catecholamine hormones. This region is highly vulnerable to hypoperfusion because of the absence of a three layer structure. All these features 
mean that differentiation between tako-tsubo and variant angina can be very difficult [11]. Both are characterized by similar clinical symptoms; however their origin is totally different. In variant angina, myocardial stunning is caused by vasospasm with subsequent loss of coronary flow reserve, which may be sometimes difficult to prove in coronary angiography $[10,12]$. An increase of sympathomimetic activity, a deficit of estrogen hormones and retention of potassium ions in extracellular spaces result in a microvascular flow deficit which plays a great role in the etiology of ABS [10].

Both analyzed cases had atypical clinical course without ST segment elevation in ECG and relatively high concentration of myocardial necrosis markers $[2,3,5-7,10,12]$. The case of M.K. was analyzed in the $52^{\text {nd }}$ volume of the Polish Cardiology Journal in 2000 - it was then considered as myocardial stunning in the course of variant angina, although it should be recognized as tako-tsubo cardiomyopathy due to typical contractility deficit, changes in ECG, and rapid augmentation of LVEF [13]. Even though tako-tsubo was first described in the 1990s, no English language studies appeared before 2000 [4]. The two cases we present suggest that recurrence of tako-tsubo is more frequent than previously thought [2, 3, 5-12]. Acute manifestation imitating acute myocardial infarction may be a part of the clinical course of the disease, which is characterized by long periods of remission and sudden onsets with doubtful prognosis of recovery [14].

Conflict of interest: none declared

\section{References}

1. Sato H, Tateishi H, Uchida T et al. Takotsubo-type cardiomyopathy due to multivessel spasm. In: Kodama K, Haze K, Hon M ed. Clinical aspect of myocardial injury: From ischemia to heart failure (in Japanese). Kagakuhyouronsya Co., Tokyo 1990: 56-64.
2. Tsuchihashi K, Ueshima K, Uchida T et al. Angina PectorisMyocardial Infarction Investigations in Japan. Transient left ventricular apical ballooning without coronary artery stenosis: A novel heart syndrome mimicking acute myocardial infarction. Angina Pectoris-Myocardial Infarction Investigations in Japan. J Am Coll Cardiol, 2001; 38: 11-18.

3. Bybee KA, Kara T, Prasad A et al. Systemic review transient left ventricular apical ballooning: a syndrome that mimics ST-segment elevation myocardial infarction. Ann Intern Med, 2004; 141: 858.

4. Kawai S, Suzuki H, Yamaguchi H et al. Ampulla cardiomyopathy ("Takotsubo" cardiomyopathy): Reversible left ventricular dysfunction with ST segment elevation. Jpn Circ J, 2000; 64: 156-159.

5. Wittstein IS, Thiemann DR, Lima JAC et al. Neurohumoral features of myocardial stunning due to sudden emotional stress. N Engl J Med, 2005; 6: 539-548.

6. Bybee KA, Prasad A, Barsness GW et al. Clinical characteristics and thrombolysis in myocardial infarction frame counts in women with transient left ventricular apical ballooning syndrome. Am J Cardiol, 2004; 94: 343-346.

7. Kurisu S, Sato H, Kawagoe T et al. Tako-tsubo-like left ventricular dysfunction with ST-segment elevation: A novel cardiac syndrome mimicking acute myocardial infarction. Am Heart J, 2002; 143: 448-455.

8. Elesber AA, Prasad A, Lennon RJ et al. Four-year recurrence rate and prognosis of the apical ballooning syndrome. J Am Coll Cardiol, 2007; 50: 448-452.

9. Nef HM, Mollmann H, Troidl Ch et al. Abnormalities in intracellular $\mathrm{Ca} 2^{+}$regulation contribute to the pathomechanism of Tako-Tsubo cardiomyopathy. Eur Heart J, 2009; 13: 2-10.

10. Nyui N, Yamanka O, Nakayama R, Sawano M, Kawai S. Tako-tsubo transient ventricular dysfunction. Jpn Circ J, 2000; 64: 715-719.

11. Dote K, Sato H, Tateishi $\mathrm{H}$ et al. Myocardial stunning due to simultaneous multivessel coronary spasms: A review of 5 cases. J Cardiol, 1991; 21: 203-214.

12. Akashi YJ, Nakazawa K, Sakakibara M et al. The clinical features of Takotsubo cardiomyopathy. QJM, 2003; 96: 563-573.

13. Wilczewski P, Glanowska G, Fudal $\mathrm{M}$ et al. Severe myocardial stunning in a female patient with variant angina: A case report. Kardiol Pol, 2000; 52: 206-210.

14. Opolski G, Kochanowski J, Torbicki A et al. The recurrence after ten years: "Mother in-law variant" of tako-tsubo syndrome. Kardiol Pol, 2010; 68: 557-561. 